Proceedings of the 2006 Winter Simulation Conference

L. F. Perrone, F. P. Wieland, J. Liu, B. G. Lawson, D. M. Nicol, and R. M. Fujimoto, eds.

\title{
PULL PRODUCTION CYCLE-TIME UNDER VARYING PRODUCT MIXES
}

\author{
Chakkaphan Athapornmongkon \\ Jochen Deuse \\ Production Systems and Industrial Engineering \\ Leonhard-Euler-Strasse 5 \\ D-44227 Dortmund, Germany
}

\author{
Chee Yew Wong \\ Cranfield University School of Management \\ Cranfield, Bedfordshire \\ MK43 0AL United Kingdom
}

\begin{abstract}
To satisfy customer requirements and simultaneously fully utilize the production machines, there is a need to determine appropriate cycle time. Especially in pull systems with varying product mixes, accurate cycle time is even more crucial. Accurate cycle time also reduces the need for buffers. When the product mix varies, especially in a pull production system with parallel flow, determination of accurate cycle time becomes problematic. Hence this paper studies the relations between cycle times and product mixes in a parallel pull production system using discrete event simulation. Experiments with varying product mixes for parallel production systems are simulated. The simulation results show that optimal cycle time is inversely proportional to product mixes, when the product mix is $50: 50$ e.g. $120 \mathrm{sec}$. should be set to each parallel machine to obtain overall cycle time of $60 \mathrm{sec}$. However, when the mixes are more than 50:50, the proportional rule is not valid.
\end{abstract}

\section{INTRODUCTION}

Production processes work efficiently if the processing tasks and their respective cycle or takt times are appropriately assigned to the designated work stations. This is extremely important, especially for the pull production system. The efficiency of the pull production system is also affected by varying product mixes, because it contributes to blockages and idleness of the machines or resources in the production or assembly lines. To dilute the effect of product mixes and optimize the utilization of the work stations, there is a need to determine a proper cycle time.

In pull systems, the customer demand rate is translated into takt time, which is used to control the pace or rhythm of the production line (Productivity Press Development Team, 2002). However, takt time can be easily determined for the serial production flow but not the flow with parallel machines. When multiple machines work in parallel to one another, their takt times cannot be easily determined as the way takt time is determined for the serial production flow.
The complexity is further increased when the parallel flow faces varying product mixes. Therefore there is a need to find ways to revise the cycle time in parallel production flows when the product mix varies.

Inappropriate takt time causes waste. Waste, such as idle machines and additional buffers, is anything that does not add any value to the products or services provided to the customers (Mottershead 2001). Waste increases throughput time and reduces resource utilization. Therefore it must be eliminated. This paper especially deals with the waste of idle resources and blockages of the production flow. It suggests a way to eliminate those wastes from the pull parallel production line.

Effective pull production requires accurate calculation of cycle time. Revising cycle time can dilute the effect of varying product mixes. In such conditions, the interaction between the product mix and resources may not be clearly visualized by a mathematical model. Instead, discrete event simulation can be used to better understand the interaction between cycle time, product mix and resource utilization. Pedgen (1995) claim that a simulation study makes it possible to predict the behavior of a complex manufacturing system by calculating the movement and other interaction of system components. Harrell (1995) also states that traditional methods like process mapping, flow charting, work analysis and linear programming are not capable of solving the complex integration problems of today. Simulation is therefore a powerful analysis tool for evaluating system design and system operation.

This paper examines how to alter takt time of parallel production lines under multiple products with varying product mixes, so that resource utilization is improved. In the next section, five experiments are simulated with single machine-parallel flow and another three experiments on multiple machine-parallel flow are simulated with varying product mixes and buffers. The experiments presented in this paper are taken as fundamental examples to show how the 'inverse proportion rule' can be applied. They are easy to understand. Even though the cases shown in this paper 
are simple, they are considered to be fundamental and can be further applied to a more complicated flows.

\section{SINGLE MACHINE-PARALLEL FLOW}

\subsection{Basic model and experiment set-up}

In this section, analyses are made to observe the parallel flow phenomenon of a simple production line. The experiments are conducted with the production line shown in Figure 1.

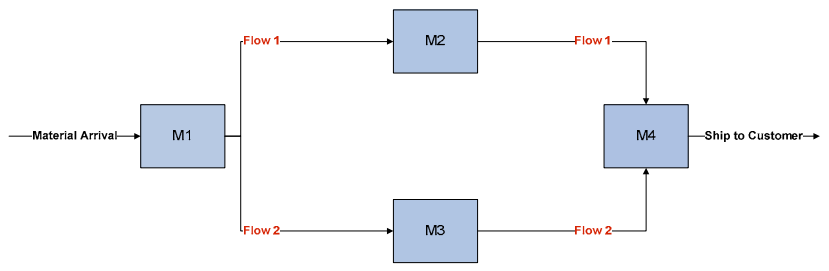

Figure 1: The Process Flow

The production flow starts with the arrival of two different types of components from different suppliers to the assembly plant. Each component is assembled into different variants. Different customers order different variants of finished goods. This causes the variation in the product mix. The arrival sequences of the different components are calculated by a scheduling algorithm before starting production. The two variants produced by machine 1 are called model A and model B. Next, model A is processed by machine 2 and model $\mathrm{B}$ is processed by machine 3 in parallel. The path going through machine 2 is named flow 1 and the path going through machine 3 is named flow 2 . These two paths bring different variants to machine 4 according to the sequenced schedule set at the beginning of the flow. Machine 4 then assembles these variants from the two parallel machines according to the sequence set. The products from machine 4 are the final products demanded by the customers. The movements along the flow and between the machines are controlled by conveyor lines. The flow described is then used to conduct the experiments.

\subsection{Result for single machine-parallel flow}

Table 1 summarizes the experiments conducted in this section. Experiments conducted in this section are varied between $50 / 50$ and $70 / 30$ in product mixes as these two mixes are used as fundamental examples to other product mixes.

The simulation results of the above five experiments for single-machine parallel flow systems are shown in table 2 . They are explained hereafter. If the demand rate required by the customers (takt time) is 60 seconds per output (cycle time of 60 seconds), the whole production line must be able to produce 60 pieces per hour.
Table 1: Experiments for Single Machine-Parallel Flow

\begin{tabular}{|r|r|r|r|}
\hline Experiment & $\begin{array}{c}\text { Product } \\
\text { Mix (A/B) } \\
\text { in \% }\end{array}$ & Buffer & $\begin{array}{c}\text { Throughput } \\
\text { time for } \\
\text { M2/M3 in } \\
\text { sec. }\end{array}$ \\
\hline 1 & $50 / 50$ & No & $60 / 60$ \\
\hline 2 & $50 / 50$ & No & $120 / 120^{*}$ \\
\hline 3 & $70 / 30$ & No & $85.7 / 200^{*}$ \\
\hline 4 & $70 / 30$ & No & $100 / 200$ \\
\hline 5 & $70 / 30$ & Yes & $85.7 / 200^{*}$ \\
\hline
\end{tabular}

* the above throughput times for M2 and M3 are calculated based on the inverse proportion rule.

$i$ this experiment is treated later in this section and the process flow is according to figure 2 .

Table 2: Simulated Results of Parallel Flow with Single Machine

\begin{tabular}{|l|r|r|r|r|r|}
\hline $\begin{array}{l}\text { Measurement } \\
\text { Factors }\end{array}$ & $\mathbf{1}$ & $\mathbf{2}$ & $\mathbf{3}$ & $\mathbf{4}$ & $\mathbf{5}$ \\
\cline { 2 - 6 } & 150 & 150 & 86 & 84 & 141 \\
\hline $\begin{array}{l}\text { Amount of } \\
\text { Finished Goods }\end{array}$ & $100 \%$ & $100 \%$ & $56.99 \%$ & $56.44 \%$ & $93.78 \%$ \\
\hline $\begin{array}{l}\text { Utilization of } \\
\text { Machine 1 }\end{array}$ & $50 \%$ & $100 \%$ & $57.45 \%$ & $65.78 \%$ & $93.76 \%$ \\
\hline $\begin{array}{l}\text { Utilization of } \\
\text { Machine 2 }\end{array}$ & $50 \%$ & $100 \%$ & $57.08 \%$ & $57.56 \%$ & $93.78 \%$ \\
\hline $\begin{array}{l}\text { Utilization of } \\
\text { Machine 3 }\end{array}$ & $100 \%$ & $100 \%$ & $57.27 \%$ & $56.22 \%$ & $93.78 \%$ \\
\hline $\begin{array}{l}\text { Utilization of } \\
\text { Machine 4 }\end{array}$ & & & & & \\
\hline
\end{tabular}

Therefore, experiment 1 is set up by limiting the throughput time of all machines to 60 seconds. 150 finished products were produced within the mentioned simulation time. The utilization of machines 1 and 4 was $100 \%$ which means they were fully utilized. However machines 2 and 3 were not fully utilized. In both cases their utilization was only $50 \%$. This shows that these two machines had spare capacities of up to $50 \%$. In other words, machines 2 and 3 had time buffers.

Experiment 2 is set up so that machines 2 and 3 are simulated at a cycle time of 120 seconds. In this experiment, the production mix is retained to $50 \%$ in both flows. Table 2 shows that the total number of finished products is equivalent to the yield resulted in the first experiment, which is 150 pieces. Since the production time of machines 2 and 3 is lengthened, the average production time is affected and therefore the lead time is higher than in the first experiment by approximately 1 minute when compared. Thus, utilization rates for all machines were $100 \%$.

The result of both experiments 1 and 2 explains that machines that work parallel to each other need higher production rates or otherwise their rates of utilization will be lower. This can clearly be seen by the percentage of machines idleness of the. A closer look at this phenomenon of parallel machines leads us to an important observation, i.e., 
the cycle times of the machines in the parallel flow were inversely proportional to the product mixes. In other words, the product mix should be inversely proportional to the demand rate set by the customer, to obtain full utilization of the machines. This 'inverse proportion rule' is as shown in the following formula:

$$
T P=\frac{T}{M_{i}}
$$

Where: $T P$ is desired throughput time

$T$ is takt time of the whole production line

$M_{i}$ is the mix in percentage of product $i$

If the demand set up by the customers is divided by the percentage of production mix, the most appropriate throughput time for each parallel machine can be obtained. Taking experiment 2 as an example, the revised cycle time according to the 'inverse proportion rule' is therefore,

$$
\frac{60 \mathrm{sec} .}{0.5}=120 \mathrm{sec}
$$

The example above shows how the balanced cycle time can be obtained. This shows that the machine capacity is increased to $100 \%$ more than its current capacity to make the flow better. In return the whole production line is $100 \%$ in utilization and no bottleneck presents in the production flow.

In order to prove the 'inverse proportion rule', the third experiment is simulated. The production mix for the third experiment is $70 \%$ in flow one and $30 \%$ in flow 2 . Hence the throughput time for machine 2 is equal to

$$
\frac{60 \mathrm{sec} .}{0.7} \approx 85.7 \mathrm{sec} .
$$

while the throughput time for machine 3 should be equal to

$$
\frac{60 \mathrm{sec} .}{0.3}=200 \mathrm{sec} .
$$

From the calculation above, the third experiment is set up. The result of this experiment is described in table 2 . Only 86 pieces of goods were produced. The utilization rates for machines 1 up to 4 were $56.99,57.45,57.08$, and 57.27 in unit of percentage, respectively. The utilization rates were low in this case, since there were blockages in the flow.

Most significant facts that should not be neglected are the blockage and the utilization rate. From the result of this third experiment, one conclusion can be made. Different product mixes affect the flow of production. Observation of machine 2 , shows that it is blocked approximately $33 \%$ of the time. Since model A was simulated at $70 \%$ of mixed proportion at flow 1, there were more variants going through flow path 1 than through flow path 2 . Furthermore, machine 4 had to produce strictly according to the set of schedule that was set in the beginning of the production. Therefore both model are processed at the machines according to the schedule set. For example, if the schedule is $\mathrm{ABAB}$ and if both models $\mathrm{A}$ and $\mathrm{B}$ are ready for machine 4 to be processed, M4 would pick model A before B. This means that when the variant flows along path 1 and reaches machine 4, it processes the model immediately as long as the machine is free.

Hence, if machine 4 has to process the model that flows on path 2 , machine 2 is then blocked, since machines 2 and 3 begin their processes in parallel. Machine 3 usually finishes processing before machine 2, due to unequal throughput time. Machine 3 finishes processing soon after passing on the processed model to the final machine 4 . This causes machine 2 to be blocked since the model processed on machine 2 cannot flow to the final machine. Usually, if the leading machine is blocked the machine that follows should be blocked as well. This explains the phenomenal effect of the flow. To check if the assumption of the 'inverse proportion rule' is correct, the throughput time on machine 2 is increased to 100 seconds. It is done to solve the effect of blockages. The analysis of the result for the fourth experiment is reported in the next paragraph.

In the fourth experiment, the throughput time of machine 3 is kept constant, since it has a high idle rate. Hence machine 3 does not cause the blockage at machine 1 . However, if its throughput time is increased, this results in a greater idleness. The effect would be the same if the throughput time of machine 2 was reduced. However the production rate of this experiment is not at the pace set. This can be proved by the average cycle time of the parallel system.

$$
\frac{(0.7 \times 100 \mathrm{sec} .)+(0.3 \times 200 \mathrm{sec} .)}{2 \text { machines }}=65 \mathrm{sec} .
$$

The calculation above proves that the cycle time of experiment 4 is equal to 65 seconds, but it is impracticable. In fact, the bottleneck in experiment 4 is shifted to the parallel machines by using this production time .

Conclusion can then be made out of the experiments conducted and reported above. As long as the production mix deviates from the ratio of 50:50, the result will always be blockages. The 'inverse proportion rule' proposed only can be used at the ratio of 50:50. However the next section shows how buffers can be used with the 'inverse proportion rule' to make the flow smooth. 


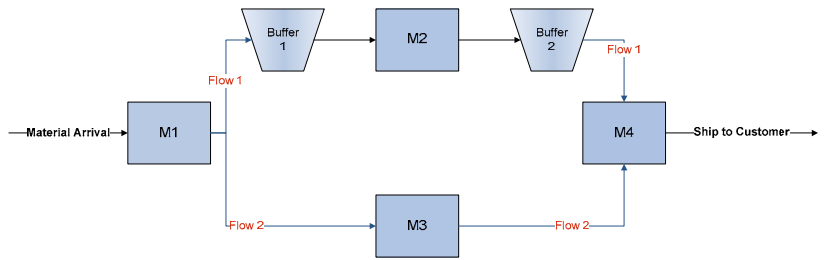

Figure 2: The Process Flow with Buffers

The weakness of the inversion rule can be countered by allocating buffers before and after the parallel machines. Buffers are used either if their priority is high or if they are needed to cope with the blockage in the pull production environment. Hence buffers should be placed between the machines as shown in figure 2. The fifth experiment is constructed by using the similar constraints to those in case 3 but with buffer. Since machine 2 has a higher throughput time than machine 1 , buffers must be placed between these two machines. As there are also some blockages between machines 2 and 4, buffers are placed there as well. In this experiment, only one buffer is placed in each of the two mentioned buffer locations. The production mix of this experiment is kept at a ratio of 70:30. All machine throughput times are kept as they were in experiment 3 .

Table 2 describes the results of experiment 5, which. has much higher utilization rates when compared to experiment 3 in numbers. Buffers help to increase the utilization. As demonstrated earlier, buffers are used to eliminate the blockages and hence increase the production rate. The result of this is the ability to satisfy customers' demand. Moreover buffers help to run machines at full capacities, if the capacities are right. Thus these satisfy the concept of the theory of constraints (Martin, 1997).

\section{MULTIPLE MACHINE-PARALLEL FLOW}

This section describes the experiments for a parallel flow system with multiple machines. The flow in figure 3 is used to conduct experiments in this section to prove that the 'inverse proportion rule' proposed earlier can be applied.

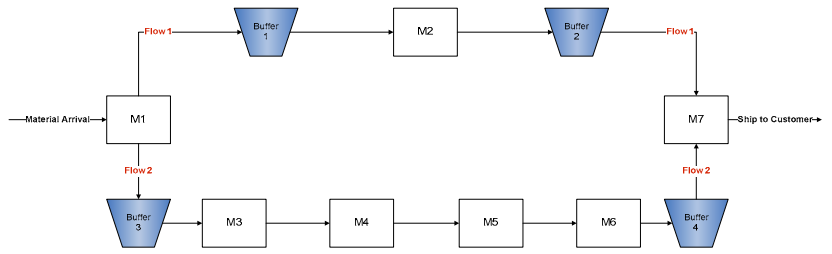

Figure 3: Parallel Production Flow

The difference between this production line and the production lines described in the previous section is the number of machines in the parallel line within the flow. In this case, the lower line of the two parallel flows consist of more than one machine. Those machines are named M3,
M4, M5 and M6, respectively. These machines work in series. Experiments are then set up in order to verify if the 'inverse proportion rule' characteristic would work in this case as well.

Table 3: Summarize the Three Experiments in this Section

\begin{tabular}{|r|r|r|r|}
\hline Experiment & $\begin{array}{c}\text { Product } \\
\text { Mix A/B } \\
\text { in \% }\end{array}$ & Buffer & $\begin{array}{c}\text { Throughput } \\
\text { time for } \\
\text { M2/M6 in } \\
\text { sec. }\end{array}$ \\
\hline 6 & $50 / 50$ & no & $60 / 60$ \\
\hline 7 & $50 / 50$ & no & $120 / 30$ \\
\hline 8 & $50 / 50$ & yes & $120 / 30$ \\
\hline
\end{tabular}

Table 3 summarizes all experiments conducted in this section. From the table, all experiments are conducted with product mix of 50:50. The reason for conducting experiments with only product mixes of 50/50 is that they represent the rest of other product mixes. If the mix of 50:50 requires buffer other mixes needed buffer as well. Therefore only the mix of 50:50 is shown in this paper.

The sixth experiment is set such that the production mix of flow 1 carries $50 \%$ of the total proportion and that of flow 2 carrying the same proportion. All machines set their throughput time, as is required by the customer, at 60 seconds. No buffer is placed between these machines in this experiment.

Table 4: Simulation Results for All Experiments in Section 3

\begin{tabular}{|l|r|r|r|}
\hline \multirow{2}{*}{ Measurement Factors } & \multicolumn{3}{|c|}{ Experiment No } \\
\cline { 2 - 4 } & \multicolumn{1}{|c|}{$\mathbf{6}$} & \multicolumn{1}{c|}{$\mathbf{7}$} & \multicolumn{1}{c|}{$\mathbf{8}$} \\
\hline Amount of Finished Goods & 100 & 60 & 150 \\
\hline Utilization of Machine 7 & $66.67 \%$ & $40 \%$ & $100 \%$ \\
\hline Utilization of Machine 2 & $33.33 \%$ & $40 \%$ & $100 \%$ \\
\hline Utilization of Machine 1 & $66.67 \%$ & $40 \%$ & $100 \%$ \\
\hline Utilization of Machine 6 & $33.33 \%$ & $40 \%$ & $100 \%$ \\
\hline
\end{tabular}

Table 4 reports the results of the sixth experiment. One hundred products were shipped. Only four machines were observed in this case, i.e., M7, M2, M1 and M6, respectively, because they play a crucial role in the production line. Utilization of these machines was poor. Two machines, M2 and M1, were blocked. Each of the machines in flow 2 had a throughput time of 60 seconds hence the total throughput time of flow 2 was 240 seconds while the machine had a flow time of 60 seconds. Parts that flowed along flow 2 blocked the flow of flow 1 , hence machine 2 is blocked. The following blockage of machine 2 was blocking machine 1 . In order to confirm that the 'inverse proportion rule' is true, the rule is then used to calculate the amount of overcapacity in terms of time. The calculation is shown below. 
Throughput time for Flow 1

$=\frac{60}{0.5}$

$=120 \mathrm{sec}$.

Throughput time for Flow 2

$=\frac{60}{0.5}$

$=120 \mathrm{sec}$.

In the seventh experiment, each machine in the lower flow is set to the cycle time of 30 seconds to satisfy the new processing time calculated according to the inverse proportion rule'. As there is only one machine in the upper flow, the cycle time for that machine is set to 120 seconds. Table 4 records the results of this experiment.

Output from this process was 60 pieces. This is due to the increment of throughput time in the parallel flow. The utilization of all machines was $40 \%$ each. Hence buffers are needed in order to make the flow better. As shown in table 4 , the inverse proportion rule helps to balance the utilization of resources in this experiment.

The eighth experiment is conducted with buffers placed along the flow. In this experiment two buffers are placed in front of machine 2 and one buffer is placed after machine 2. The result can be learned from table 4 . All the machines were fully utilized in this case and the output of 150 pieces was obtained.

To summarize, the 'inverse proportion rule' works with both types of production lines which have been dealt with in the earlier section. However buffers are needed for cases that show a high variation between the product mixes from different product types.

The multiple machine-parallel flow when compared with the single-machine parallel flow can be viewed as, , an extended application of the latter. More machines (M3 and M6) are included in the same flow as illustrated in figure 3. As both cases share common characteristic the 'inverse proportion rule' can then be applied.

However, the result is not optimal in the case that product mix is higher than $50 / 50$. This is due to the fact that different numbers of types of products are released into the production line. The effect is exaggerated if the pull production system is used. Therefore buffer must be used to lessen this effect.

\section{CONCLUSION}

In a pull production environment, production line should be flexible enough while fully utilizing all resources. However, most of the production lines produce more than one type of products. Therefore scheduling of the mixes plays a significant role in the utilization of machines. This paper suggests a way of limiting the throughput or cycle time of the resources by producing mixes in the parallel flow through the inverse proportion rule. The rule helps to design or guide engineers to achieve the balanced pull production line.

The 'inverse proportion rule' is proposed as a guideline to benchmark the takt time that helps to achieve full utilization of resources in the pull production environment. The effect of the high mix variation between the product types prevents the 'inverse proportion rule' from being optimal. However, by adding buffers in addition to applying the 'inverse proportion rule', the production resources are found to be fully utilized.

With severe production mixes, buffers must be used in concurrence with the rule in order to eliminate all the blockages and idleness effects.

The cycle time generated from the inverse proportional rule together with buffers can help to dilute the effect of parallel processing machines. However, the cycle time calculated on the basis of the 'inverse proportion rule' only includes the effect of product mix and takt time based on the customer demand at a stable stage of production. Other parameters, such as down time, amount of defects, and so on, are not included and therefore may affect the result and the proposed rule.

\section{REFERENCES}

Harrell, C., Tumay, K. 1995. Simulation made easy: a manager's guide. Institute of Industrial Engineers. ISBN 0-89806-136-9.

Martin, D.P. 1997. How the law of unanticipated consequences can nullify the theory of constraints: The case for balanced capacity in a semiconductor manufacturing line, Advanced Semiconductor Manufacturing Conference and Workshop 1997.

Mottershead, D 2001. Introducing lean manufacturing at ESI. Portland International Conference. Volume: 1. 29 July-2 Aug. 2001 Pages:448 vol.1.

Pedgen, C.D., Shannon, R.E. \& Sadowski R.P. 1995. Introduction to Simulation Using SIMAN (2. ed.) McGraw-Hill, Inc. ISBN 0-07-049320-0.

Productivity Press Development Team 2002. Standard Work for the Shopfloor. ISBN: 1-56327-273-3. Productivity Press 2002.

\section{AUTHOR BIOGRAPHIES}

CHAKKAPHAN ATHAPORNMONGKON is currently a PhD student in the Department of Production Systems and Industrial Engineering at Dortmund University, Germany. He received M.Sc from Linköping Universitet, Sweden, in Industrial Engineering and Management. His areas of research include the modeling and simulation, optimization, production system, and maintenance. He also had industrial experiences in supply chain and logistic management at Siam City Cement Public Company Lim- 
ited. His e-mail address is <chakkaphan@aps.mb. uni-dortmund.de>.

JOCHEN DEUSE is Professor at Department of Production Systems and Industrial Engineering in Dortmund University, Germany. He received Dip. Ing (Master Degree Equivalent) in Engineering Technology and Production Managements from Dortmund University, Germany and Dr. Ing (Ph.D equivalent) from Aachen Technical University, Germany. He has over five years of experience in managerial position at Robert Bosch Australia. His current research interests include Lean Production, Digital Manufacturing, and strategic production planning. His e-mail address is<deuse@aps.mb.uni-dortmund.de>.
CHEE YEW WONG is a research fellow from Cranfield University School of Management. He completed his Ph.D in Operations and Supply Chain Management from Aalborg University in Denmark, and M.Sc in Industrial Engineering and Management from Linkoping University in Sweden. In Denmark, he used to teach modeling and simulation, management information system, and supply chain management. He has over five years industrial experiences in design and supply chain management at ABB Sweden/Malaysia. Currently, his main research areas are supply chain integration strategy, supply chain planning and coordination, and inter-organizational process alignment. His email address is <hee.wong@cranfield. ac.uk> 